This item was submitted to Loughborough's Research Repository by the author.

Items in Figshare are protected by copyright, with all rights reserved, unless otherwise indicated.

\title{
Determining effective subject-specific strength levels for forward dives using computer simulations of recorded performances
}

PLEASE CITE THE PUBLISHED VERSION

\section{PUBLISHER}

(C) Elsevier

\section{VERSION}

AM (Accepted Manuscript)

\section{LICENCE}

CC BY-NC-ND 4.0

\section{REPOSITORY RECORD}

King, Mark A., Pui W. Kong, and Maurice R. Yeadon. 2019. "Determining Effective Subject-specific Strength Levels for Forward Dives Using Computer Simulations of Recorded Performances”. figshare. https://hdl.handle.net/2134/5825. 
This item was submitted to Loughborough's Institutional Repository (https://dspace.lboro.ac.uk/) by the author and is made available under the following Creative Commons Licence conditions.

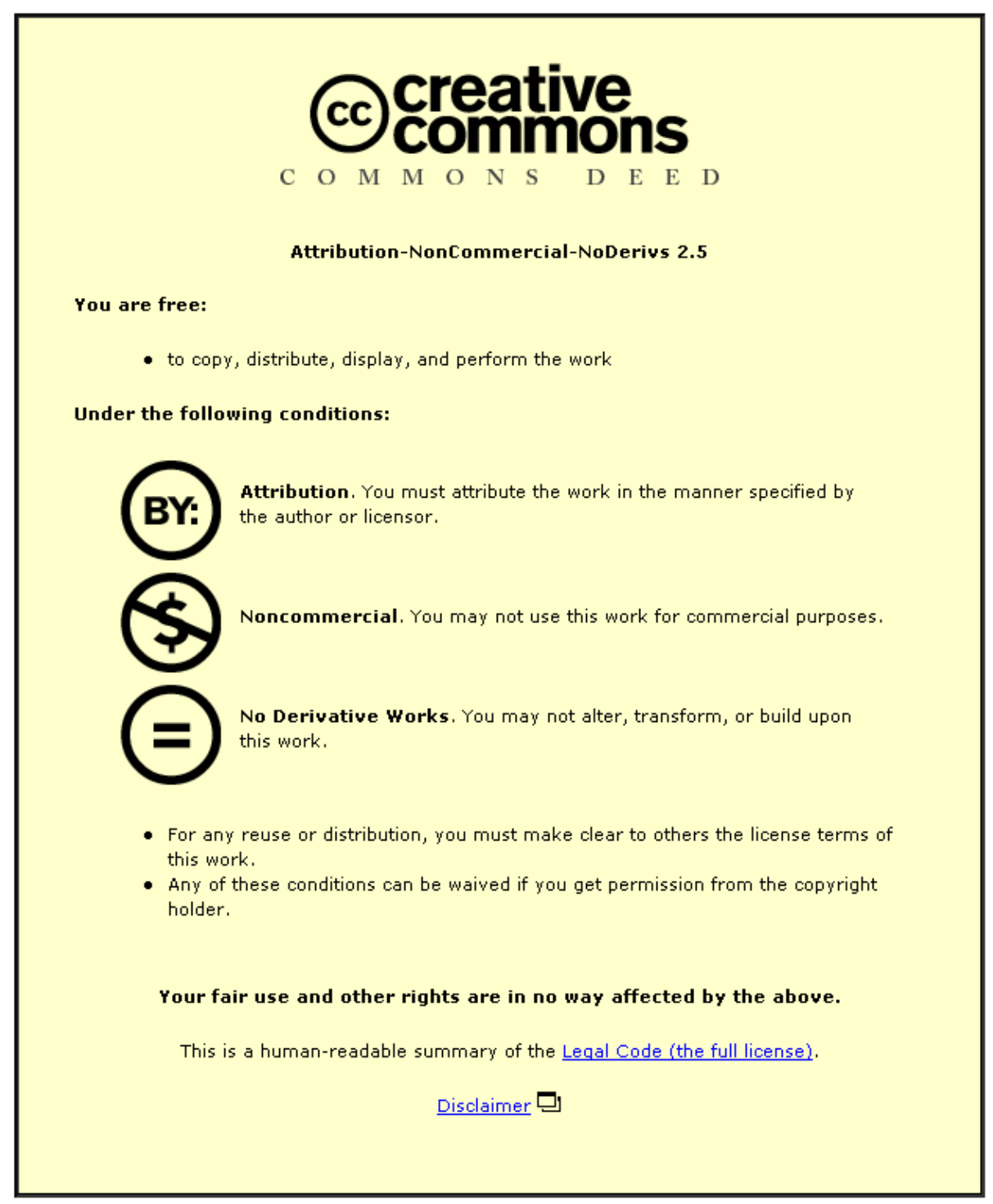

For the full text of this licence, please go to: http://creativecommons.org/licenses/by-nc-nd/2.5/ 


\title{
Determining effective subject-specific strength levels for forward dives using computer simulations of recorded performances
}

\author{
${ }^{1}$ Mark A. King, ${ }^{2}$ Pui W. Kong and ${ }^{1}$ Maurice R. Yeadon \\ ${ }^{1}$ School of Sport and Exercise Sciences, Loughborough University, Loughborough LE11 3TU, UK. \\ ${ }^{2}$ National Institute of Education, Singapore, 1 Nanyang Walk, Singapore 637616.
}

\begin{abstract}
This study used optimisation procedures in conjunction with an 8-segment torque-driven computer simulation model of the takeoff phase in springboard diving to determine appropriate subjectspecific strength parameters for use in the simulation of forward dives. Kinematic data were obtained using high-speed video recordings of performances of a forward dive pike (101B) and a forward $2 \frac{1}{2}$ somersault pike dive $(105 \mathrm{~B})$ by an elite diver. Nine parameters for each torque generator were taken from dynamometer measurements on an elite gymnast. The isometric torque parameter for each torque generator was then varied together with torque activation timings until the root mean squared (RMS) percentage difference between simulation and performance in terms of joint angles, orientation, linear momentum, angular momentum, and duration of springboard contact was minimised for each of the two dives. The two sets of isometric torque parameters were combined into a single set by choosing the larger value from the two sets for each parameter. Simulations using the combined set of isometric torque parameters matched the two performances closely with RMS percentage differences of $2.6 \%$ for $101 \mathrm{~B}$ and $3.7 \%$ for $105 \mathrm{~B}$. Maximising the height reached by the mass centre during the flight phase for $101 \mathrm{~B}$ using the combined set of isometric parameters and by varying torque generator activation timings during takeoff resulted in a credible height increase of $38 \mathrm{~mm}$ compared to the matching simulation. It is concluded that the procedure is able to determine appropriate effective strength levels suitable for use in the optimisation of simulated forward dive performances.
\end{abstract}

\section{Introduction}

Computer simulation models of various sports movements have been developed in order to investigate the mechanics of optimum performance (Alexander, 1990; King and Yeadon, 2004; Koh et al., 2003). Crucial to the development of such models is that they must be accurate representations of the biomechanical systems being studied. To assess the accuracy of a simulation model and show that it is sufficiently complex to represent the biological system, subject-specific values of inertia, strength and visco-elastic parameters are required so that the output of the model can be compared to recorded performances by the same subject in a quantitative manner (Yeadon and King, 2002). Subject-specific strength parameters are difficult to determine and have typically been calculated from isovelocity torque measurements on the subject over a range of joint angles and angular velocities at each joint (King and Yeadon, 2002, King et al., 2006; Mills et al., 2008). The limitations of this technique are that it requires access to the subject and it can be difficult for the subject to produce maximal torque values during each trial on the dynamometer.

Recently, torque-driven computer simulation models have been used to provide insights into springboard diving takeoff techniques. Cheng and Hubbard (2004) used a two-segment model with theoretically assigned muscle parameters to optimise knee extension strategies for maximum jump height from a springboard. The simplicity of this model (two segments) and the assumptions made in choosing model parameters (theoretically assigned) limit its potential use for predicting realistic diving performances. Sprigings and Miller (2004) used a five-segment model with velocity-dependent torque actuators to investigate springboard takeoffs for reverse dives. This model could not reproduce accurately the takeoff movements of an elite diver and the authors acknowledge that the model may have been limited by the 
simplicity of the torque activation profiles used for reverse dives. Alternatively the torque parameters used in the model were not specific to the elite diver and therefore might not have been representative of the diver's strength capability.

In order for a simulation model of springboard diving to be capable of matching an actual performance it is necessary for the model to be sufficiently complex and sufficiently strong. In order for such a model to be used subsequently to optimise diving technique it is also necessary that the model is not too strong. The aim of this study was to present a novel method for obtaining effective subject-specific strength parameters based on simulations of recorded performances and appropriate for simulating forward dives. Previous research with torque-driven simulation models with maximal voluntary torque functions based on dynamometer measurements indicates that for limiting movements full activation is achieved at a number of joints (King and Yeadon, 2004; Wilson et al., 2007). It is expected therefore that the proposed method will identify effective maximal joint torque levels for the types of skills used in their determination.

\section{Method}

A planar 8-segment computer simulation model of a springboard and a diver with 8 joint torque generators ( 4 extensor, 4 flexor) was developed (Figure 1) to investigate diving takeoff techniques in the forward dive group. The springboard was modelled so as to allow vertical, horizontal and rotational movements (Yeadon et al., 2006b). The diver was represented by an 8-segment linked system comprising head, upper arm, lower arm, trunk, thigh, shank and a two-segment foot. Wobbling masses were included within the trunk, thigh and shank segments to represent soft tissue movement during takeoff. It was not clear whether such wobbling masses were necessary to accurately model springboard diving so they were included with a view to investigating this. The foot-springboard interface was modelled using three pairs of parallel and perpendicular damped springs acting at the toe, metatarsalphalangeal (MTP) joint and heel. There were extensor and flexor torque generators at the ankle, knee, hip and shoulder joints plus a torsional spring at the MTP joint. The elbow $\left(\theta_{\mathrm{e}}\right)$ and the head $\left(\theta_{\mathrm{d}}\right)$ angles were driven by joint angle time histories calculated from video recordings of diving performances described below. The 8-segment model was used to simulate the board contact phase plus the first $100 \mathrm{~ms}$ of flight.

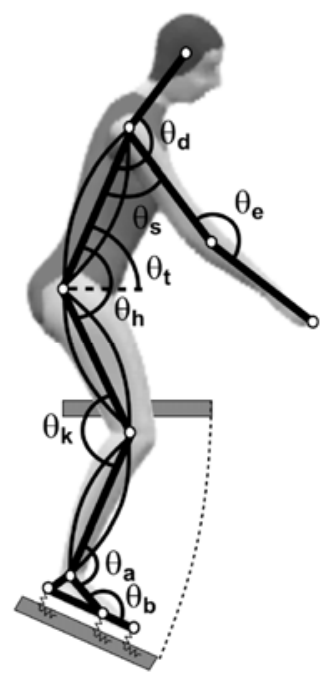

Figure 1. Planar simulation model of a springboard and an 8-segment diver with 4 extensor torque generators, 4 flexor torque generators acting at the ankle, knee, hip and shoulder joints, a ball extensor torsional spring, wobbling mass segments in the shank, thigh and trunk and angle driven neck and elbow joints. 
For each torque generator, the properties of muscle and tendon were modelled using a rotational muscle-tendon complex comprising a contractile element and a series elastic element. The torque $\mathrm{T}$ exerted at a joint was calculated as the product of the activation level $\mathrm{a}_{\mathrm{t}}$ and the maximum voluntary torque defined by a nine-parameter function of contractile element angle and angular velocity (Yeadon et al., 2006a; King et al., 2006). Specifically a six parameter function $f(\omega)$ was used to describe the torque - angular velocity relationship multiplied by the isometric torque parameter $\mathrm{T}_{\text {iso }}$ (Yeadon et al., 2006a) and a two-parameter quadratic function $\mathrm{f}(\theta)$ for the torque-angle relationship (King et al., 2006) as in equation (1).

$$
\mathrm{T}=\mathrm{a}_{\mathrm{t}} \cdot \mathrm{T}_{\text {iso }} \cdot \mathrm{f}(\omega) \cdot \mathrm{f}(\theta)
$$

The activation level of each torque generator was allowed to ramp up or down using a quintic function with zero first and second derivatives at the endpoints (Yeadon and Hiley, 2000). In particular the extensors were allowed to ramp up from a low initial level and ramp down towards the end of the board contact phase (Figure 2a). In contrast the flexors were allowed to ramp down from an initial level and then ramp up towards the end of the board contact phase to prevent hyper-extension (Figure $2 \mathrm{~b}$ ). The shoulder flexor was regarded as an "extensor" in this context since it was responsible for increasing the shoulder joint angle (Figure 1) and the shoulder extensor was regarded as a "flexor". Six parameters were required to specify the timing and level of activation for each torque generator (two start times, two ramp durations, two activation levels), giving a total of 48 parameters (Figure 2). The values of the six parameters per torque generator were allowed to vary within sensible ranges so that the general shape of each profile was maintained along with the minimum time for the activation ramping duration set at $100 \mathrm{~ms}$ (Duncan and McDonagh, 2000) for a change from 0 to 1 (or 1 to 0 ) and the maximum initial activation level at touchdown was set at $50 \%$ (Horita et al., 2002). For the torsional spring at the MTP joint the extensor torque was equal to $-\mathrm{k}_{\mathrm{s}}\left(\theta_{\mathrm{b}}-\theta_{\text {flat }}\right) \mathrm{b}_{\text {act }}$ where $\mathrm{k}_{\mathrm{s}}=$ torsional spring stiffness parameter; $\theta_{\mathrm{b}}=$ MTP angle (see Figure 1); $\theta_{\text {flat }}=$ MTP angle when the foot is flat and $b_{\text {act }}=$ constant activation level (range 0-1).

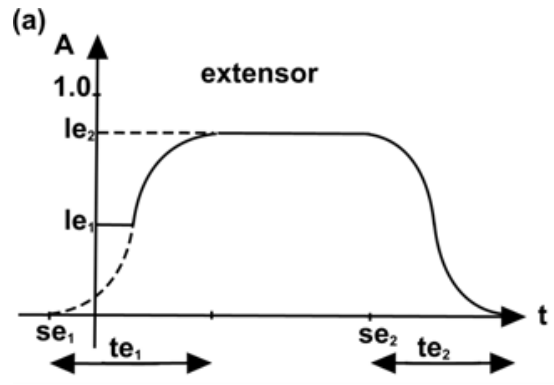

(b)

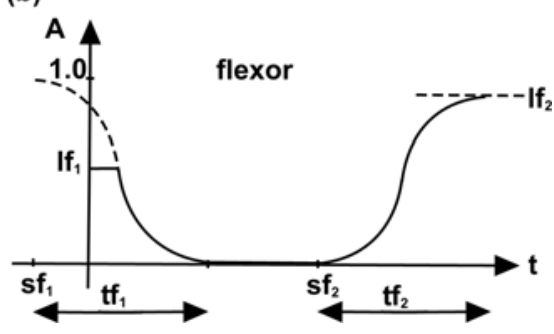

Figure 2. Activation profile of torque generators: (a) the extensor ramps up from a low initial level to a maximum level and then ramps down towards the end of takeoff; (b) the flexor ramps down from a high initial level and then ramps up towards the end. Each torque generator requires six parameters: 1) initial starting time $\left.\left(\mathrm{se}_{1}, \mathrm{sf}_{1}\right), 2\right)$ ramping duration $\left(\mathrm{te}_{1}\right.$, $\left.\mathrm{tf}_{1}\right)$, 3) final starting time $\left.\left(\mathrm{se}_{2}, \mathrm{sf}_{2}\right), 4\right)$ ramping duration $\left.\left(\mathrm{te}_{2}, \mathrm{tf}_{2}\right), 5\right)$ initial activation level $\left(\mathrm{le}_{1}\right.$, $\mathrm{lf}_{1}$ ) and 6) maximum (for extensor) or final (for flexor) activation level $\left(\mathrm{le}_{2}, \mathrm{lf}_{2}\right)$. 
The FORTRAN code implementing the torque-driven model was generated by the Autolev 3.4 ${ }^{\mathrm{TM}}$ software package based on Kane's method of formulating equations of motion (Kane and Levinson, 1985). There were 18 degrees of freedom in the model: nine defining wobbling mass displacement (Yeadon et al., 2006b), two defining foot position, one defining trunk orientation $\theta_{\mathrm{t}}$, five defining joint angles $\left(\theta_{\mathrm{b}}, \theta_{\mathrm{a}}, \theta_{\mathrm{k}}, \theta_{\mathrm{h}}, \theta_{\mathrm{s}}\right)$, and one defining vertical springboard displacement (Figure 1). The input to the model included: 1) initial conditions at touchdown comprising the vertical springboard displacement and velocity, the diver's foot position, mass centre velocity, trunk orientation and angular velocity, and 2) torque generator activation time histories. The output from the model comprised the time histories of the springboard displacement, angle and angular velocity at each joint, trunk orientation, mass centre velocity and whole-body angular momentum about the mass centre.

The forward dive pike (101B, FINA 2009) and forward 21/2 somersault pike (105B) were selected for the matching since these dives have minimal (101B) and maximal (105B) angular momentum requirements in the forward dive group. An elite female diver (mass = $64.1 \mathrm{~kg}$, height $=1.68 \mathrm{~m}$ ) competing at junior international level provided informed consent as approved by the Loughborough University Ethical Advisory Committee. Subject-specific segmental inertia parameters were determined from 95 anthropometric measurements using the mathematical model of Yeadon (1990). Kinematic data on the two diving performances from a one-metre springboard were obtained from high speed video recordings (Yeadon et al., 2006b). Joint torque time histories on the two diving performances were calculated using an angle driven model (Yeadon et al., 2006b, Yeadon and King, 2008). Springboard parameters and visco-elastic parameters of the wobbling masses and the foot-springboard interface were determined using an angle-driven model (Yeadon et al., 2006b). The nine torque parameters per torque generator were taken from an elite male gymnast (calculated from dynamometer torque measurements (Mills et al., 2008)). The isometric strength parameter for each torque generator (which governed the overall torque magnitude) was then allowed to vary (along with the MTP torsional spring stiffness parameter) in the matching of each dive. It was assumed that each torque generator reached full activation and so the activation levels $\mathrm{le}_{2}$ and ${ }_{1} f_{2}$ were set equal to 1.0 (see Figure 2 ) and $b_{a c t}=1.0$. This process applied to the two dives gave two sets of isometric parameters plus MTP joint stiffness from which the higher parameter of each pair was chosen to give a common set of subject-specific strength parameters.

The matching process consisted of minimising a difference score $\mathrm{S}$ which calculated the RMS difference between a simulation and the performance in terms of $S_{1}$ : joint angles, $S_{2}$ : trunk orientation, $\mathrm{S}_{3}$ : whole body linear momentum, $\mathrm{S}_{4}$ : whole body angular momentum about the mass centre, $S_{5}$ : duration of springboard contact for each dive (equation (2)). All five components of the difference score were considered to be equally important when objectively quantifying the difference between a simulation and performance and so were equally weighted (equation (2)). For the difference scores $\mathrm{S}_{1}$ and $\mathrm{S}_{2}$ which were calculated in degrees, $1^{\circ}$ was considered to be comparable to $1 \%$ for other scores and therefore $1^{\circ}$ was counted as $1 \%$. Anatomical constraints on the knee and ankle joints during takeoff and the first $100 \mathrm{~ms}$ of flight were used to ensure that simulations were realistic. The knee and ankle joint angles (Figure 1) were not allowed to exceed the range observed in the recorded dives either at takeoff or during the first $100 \mathrm{~ms}$ of the simulated flight phase. If a simulation resulted in any of the constraints being violated, penalties were added to the difference score for the simulation (equation (2)).

$$
\mathrm{S}=\sqrt{\frac{1}{5} \sum_{\mathrm{i}=1}^{5} \mathrm{~S}_{\mathrm{i}}^{2}}+\text { penalties }
$$


In particular the joint angles score $\mathrm{S}_{1}$ was the RMS difference in degrees between the time histories of five simulation joint angles (sja) and the performance joint angles (pja) calculated from video recordings until the point of takeoff from the springboard (n time intervals): $\quad S_{1}=\sqrt{\frac{1}{5} \sum_{j a=1}^{5} \frac{1}{n} \sum_{\text {time=1 }}^{n}\left(\theta_{\text {sja }}-\theta_{\text {pja }}\right)^{2}}$. The orientation score $S_{2}$ was calculated as the RMS difference in the trunk angle $\left(\theta_{\mathrm{t}}\right)$ time history until the point of takeoff from the springboard: $S_{2}=\sqrt{\frac{1}{n} \sum_{\text {time }=1}^{\mathrm{n}}\left(\theta_{\mathrm{st}}-\theta_{\mathrm{pt}}\right)^{2}}$. The linear momentum score $\mathrm{S}_{3}$ was the RMS percentage difference in mass centre horizontal $\left(\mathrm{V}_{\mathrm{h}}\right)$ and vertical velocities $\left(\mathrm{V}_{\mathrm{v}}\right)$ at takeoff: $\mathrm{S}_{3}=100 \sqrt{\frac{1}{2} \sum_{\text {vel. }=h, \mathrm{v}}\left(\left(\mathrm{V}_{\mathrm{sv}}-\mathrm{V}_{\mathrm{pv}}\right) / \mathrm{V}_{\mathrm{pv}}\right)^{2}}$. The angular momentum score $\mathrm{S}_{4}$ was the percentage difference in the angular momentum $\mathrm{H}$ at takeoff: $\mathrm{S}_{4}=100 \sqrt{\left(\left(\mathrm{H}_{\mathrm{s}}-\mathrm{H}_{\mathrm{p}}\right) / \mathrm{H}_{\mathrm{p}}\right)^{2}}$. The duration of springboard contact score $\mathrm{S}_{5}$ was the percentage difference in takeoff duration $\mathrm{T}$ : $S_{5}=100 \sqrt{\left(\left(T_{s}-T_{p}\right) / T_{p}\right)^{2}}$.

Initial kinematic conditions for the two dives at touchdown were obtained from the recorded diving performances. In addition to the 40 torque activation parameters (the other 8 activation parameters were set to full activation), 8 isometric torque parameters (4 flexor and 4 extensor) and one MTP joint stiffness parameter, the initial trunk angle $\left( \pm 1^{\circ}\right)$ and angular velocity $\left( \pm 1 \mathrm{rad} \mathrm{s}^{-1}\right)$ were also allowed to vary in the matching process to compensate for errors in the kinematic data. For each dive, 51 parameters were varied until the best match between the simulation and the performance was found by minimising the difference score $\mathrm{S}$ using the Simulated Annealing optimisation algorithm (Corana et al., 1987) which typically ran for 3 days with up to 50,000 simulations. No penalties were incurred for joint constraint violations in either of the matching simulations. Once a common set of maximum isometric parameters plus MTP joint stiffness had been determined these parameters were fixed. The matching process was then repeated for each dive using the common set of isometric parameters with the maximum activation to each torque generator / MTP torsional spring $\left(\mathrm{le}_{2}\right.$, $\mathrm{lf}_{2}$ and $\mathrm{b}_{\mathrm{act}}$ ) allowed to vary up to 1.0 for the joints which had lower isometric parameters in the initial matching. This gave two new matching simulations which used a common set of torque parameters. It was expected that the same level of matching would be achieved using this combined parameter set since a higher isometric torque value would be compensated by a sub-maximal activation level.

After satisfactory matching, the model was applied to optimise the takeoff technique of 101B for maximum dive height. This was done by re-optimising the torque activation parameters to search for a solution that would produce maximum dive height while keeping the same board clearance (horizontal) distance and rotation potential as in the matching simulation $($ rotation potential $=$ angular momentum at takeoff $\times$ flight time). The initial trunk angle and angular velocity were the same as in the matching simulation. Penalty scores were used to ensure that the horizontal board clearance and rotation potential were the same as the matching and to prevent joint hyper-extension; no penalties were incurred in the optimised simulation.

The necessity to include wobbling mass segments within the diver model was assessed by repeating the matching simulation optimisation for 105B with the stiffness and damping parameters for the three wobbling mass segments increased by a factor of 500 .

In order to evaluate the effect of a matching simulation on the performance of a dive the flight phase performance was calculated using an angle-driven simulation model of aerial movement (Yeadon et al., 1990) based on the takeoff characteristics of the simulation comprising trunk orientation, linear momentum, and angular momentum, together with joint 
angle time histories obtained from the video recordings. The takeoff configuration was merged into the configuration of the performance over the first $100 \mathrm{~ms}$ of flight using a quintic function (Yeadon and Hiley, 2000).

\section{Results}

The initial matching simulations were in good agreement with the two recorded performances with difference scores of $2.6 \%$ for $101 \mathrm{~B}$ and $3.7 \%$ for $105 \mathrm{~B}$. Most of the isometric parameters were taken from the matching of 105B (higher value chosen for each pair), with only the isometric hip flexion torque parameter and ankle dorsi-flexion torque parameter being taken from the matching of 101B (Table 1).

Table 1. Comparison of isometric parameters $[\mathrm{Nm}]$ determined from the initial matching of the two dives

\begin{tabular}{lccc}
\hline \multicolumn{1}{c}{ isometric parameter } & 101B & $105 \mathrm{~B}$ & combined \\
\hline knee extension & 142 & 182 & 182 \\
knee flexion & 138 & 143 & 143 \\
hip extension & 257 & 318 & 318 \\
hip flexion & 192 & 180 & 192 \\
shoulder extension & 26 & 109 & 109 \\
shoulder flexion & 59 & 63 & 63 \\
ankle plantar flexion & 216 & 273 & 273 \\
ankle dorsi flexion & 51 & 44 & 51 \\
MTP stiffness [Nm/rad] & 35 & 68 & 68 \\
\hline
\end{tabular}

Table 2. Contributions to difference scores for the two matching simulations

\begin{tabular}{llll}
\hline \multicolumn{1}{c}{ score } & & $101 \mathrm{~B}$ & $105 \mathrm{~B}$ \\
\hline joint angles: & $\mathrm{S}_{1}$ & $4.6^{\circ}$ & $5.5^{\circ}$ \\
orientation & $\mathrm{S}_{2}$ & $1.2^{\circ}$ & $2.0^{\circ}$ \\
linear momentum & $\mathrm{S}_{3}$ & $1.5 \%$ & $1.8 \%$ \\
angular momentum & $\mathrm{S}_{4}$ & $0.2 \%$ & $0.4 \%$ \\
time of contact & $\mathrm{S}_{5}$ & $3.0 \%$ & $5.6 \%$ \\
average score & $\mathrm{S}$ & $2.6 \%$ & $3.7 \%$ \\
\hline
\end{tabular}

where $\mathrm{S}_{1}$ consists of components for 101B: $5.0^{\circ}, 6.3^{\circ}, 3.1^{\circ}, 3.8^{\circ}, 4.3^{\circ}$ and for 105B: $5.1^{\circ}$, $10.2^{\circ}, 3.7^{\circ}, 2.3^{\circ}, 1.5^{\circ}$ for the ball, ankle, knee, hip and shoulder joints respectively.

Table 3. Comparison of takeoff variables for the two matching simulations

\begin{tabular}{ccccc}
\hline variable & \multicolumn{2}{c}{$101 \mathrm{~B}$} & \multicolumn{2}{c}{$105 \mathrm{~B}$} \\
& performance & simulation & performance & simulation \\
\hline$\theta_{\mathrm{t}}$ & $69^{\circ}$ & $67^{\circ}$ & $14^{\circ}$ & $20^{\circ}$ \\
$\mathrm{V}_{\mathrm{cmx}}$ & 0.87 & 0.86 & 1.33 & 1.33 \\
$\mathrm{~V}_{\mathrm{cmz}}$ & 4.75 & 4.81 & 4.39 & 4.50 \\
$\mathrm{AM}$ & 17.1 & 17.1 & 58.9 & 58.7 \\
\hline
\end{tabular}

Note: $\theta_{\mathrm{t}}=$ trunk orientation; $\mathrm{v}_{\mathrm{cmx}}=$ horizontal velocity of the mass centre $\left[\mathrm{ms}^{-1}\right] ; \mathrm{v}_{\mathrm{cmz}}=$ vertical velocity of the mass centre $\left[\mathrm{ms}^{-1}\right] ; A M=$ whole-body angular momentum $\left[\mathrm{kg} \mathrm{m}^{2} \mathrm{~s}^{-1}\right]$. 
Using the single set of isometric torque parameters (Table 1) and allowing maximum activations to be less than one resulted in two finalised matching simulations which were also in good agreement with the recorded performances with difference scores of $2.6 \%$ for $101 \mathrm{~B}$ and $3.7 \%$ for $105 \mathrm{~B}$ as expected (Tables 2 and 3). $101 \mathrm{~B}$ was matched marginally better than $105 \mathrm{~B}$ in terms of all five components of the difference score and this may well be due to $105 \mathrm{~B}$ being a more demanding and complex dive. Linear momentum and angular momentum at board takeoff (Table 3) were closely matched while the joint angle time histories deviations although quite similar (Figure 5) were harder to match. In particular the knee, hip and shoulder joints tended to be matched quite closely while the ball and ankle joints were matched less closely. The reason for this may be that the angle changes at the knee, hip and shoulder joints are more important for the matching of the linear momentum and angular momentum than the matching of the ball and ankle joint kinematics. The graphics sequences in Figures 3 and 4 show close correspondence in both the takeoff and flight phases between each matching simulation and the corresponding recorded performance of each dive. The joint torque time histories in the matching simulation (101B) were comparable in magnitude but smoother than the torque time histories obtained using the angle-driven model (Figure 6) which were noisy due to errors in the joint angle estimates obtained via manual digitisation (Yeadon and King, 2008).

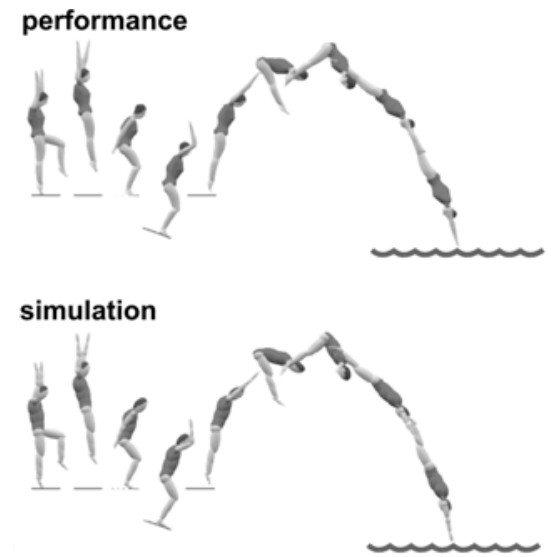

Figure 3. Comparison of performance and matching simulation of a forward dive pike (101B) (with additional $0.6 \mathrm{~m}$ horizontal spacing between contact phase graphics and $0.4 \mathrm{~m}$ between flight phase graphics).

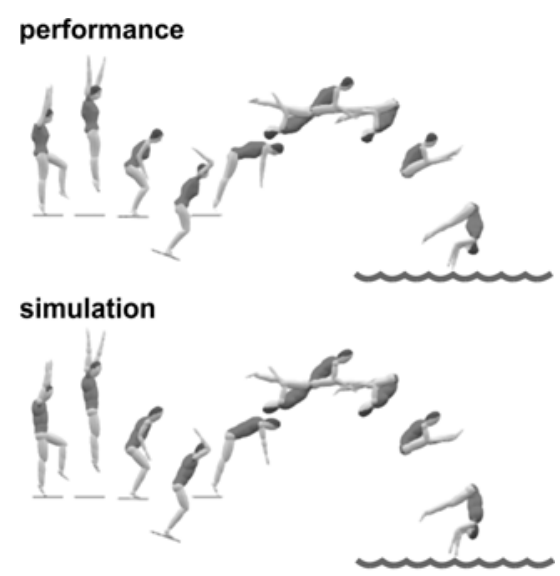

Figure 4. Comparison of performance and matching simulation of a forward two and one-half somersault pike (105B) (with additional $0.6 \mathrm{~m}$ horizontal spacing between contact phase graphics and $0.4 \mathrm{~m}$ between flight phase graphics). 


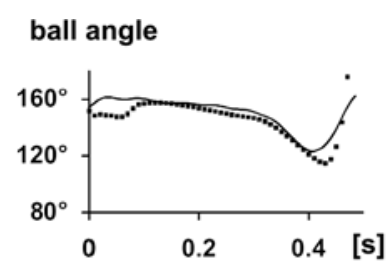

ankle angle

knee angle
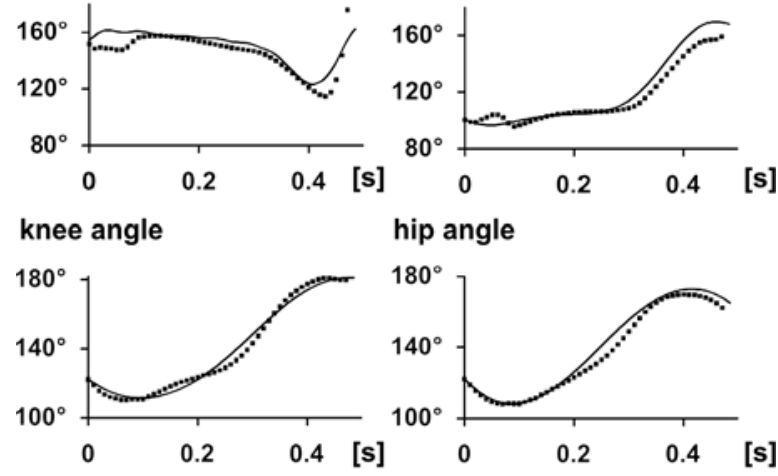

hip angle

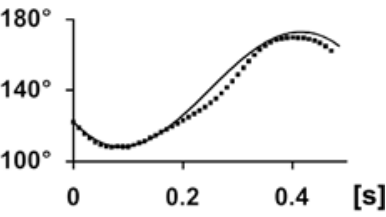

shoulder angle

trunk orientation
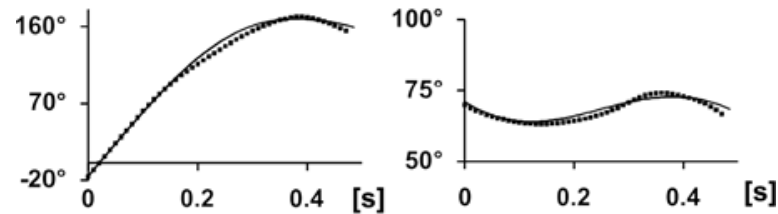

horizontal $\mathrm{cm}$ position [m]

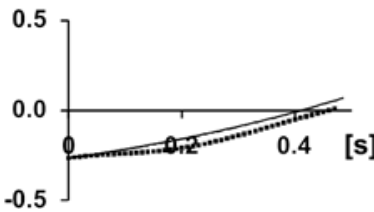

vertical $\mathrm{cm}$ position [m]

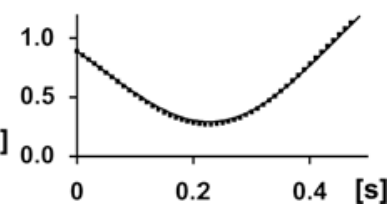

Figure 5. Comparison of key kinematic variables for the forward dive pike (101B) performance (solid line) and the matching simulation (square markers).
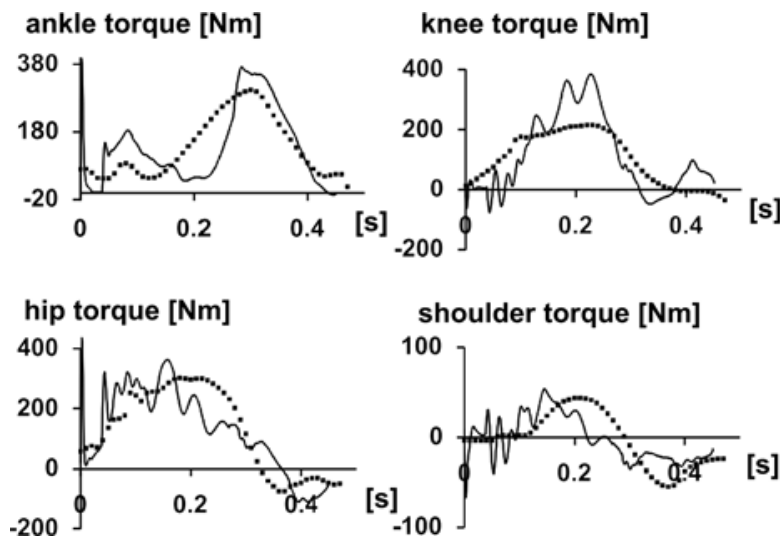

Figure 6. Torque time histories of the forward dive pike (101B) calculated from the angledriven model (solid line) and the torque-driven model matching simulation (square markers).

In the optimisation for maximum dive height, there was a $38 \mathrm{~mm}$ increase in height compared with the 101B matching simulation with only small differences in the activation time histories (Figure 7). One slight difference was for the activating timing of the ankle plantar flexors (Figure 7) which ramped up later in the optimisation. As a consequence it could be speculated that delaying the activation of the ankle plantar flexors appears to be important to the generation of vertical velocity. Furthermore the joint angle time histories of the matching and the optimised simulations showed only small differences and mass centre positions during board contact were indistinguishable from those shown in Figure 5. The 
similar torque activation profiles and kinematics are probably due to the actual performance being close to optimal and the need to keep the horizontal board clearance and rotation potential the same.
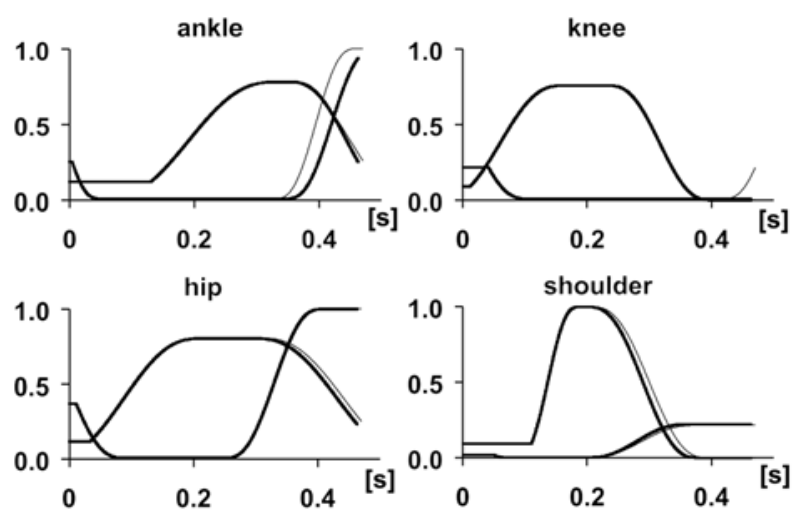

Figure 7. Comparison of the activation time histories for the matching simulation of 101B (thin line) and the optimised solution for height (thick line).

When the matching optimisation for 105B was repeated with increased stiffness and damping parameters for the wobbling masses, the optimum matching simulation was very similar to the original both in terms of the activation time histories used and the resulting difference score which changed by less than $0.2 \%$.

\section{Discussion}

The close match between simulation and performance in terms of diver kinematics and joint torques used during the takeoff phase indicates that the model incorporates the necessary features of springboard diving and successfully reproduces actual takeoff movements. The small differences between matching simulations and performance during the takeoff phase result in a good match of each performance during flight. As a consequence the level of model complexity is appropriate to allow studies into technique and optimum performance in forward dives (Yeadon and King, 2008).

The calculated effective joint torque strength of the model is appropriate for simulating forward dives in that it was sufficient to permit a good matching and allowed only a modest $(38 \mathrm{~mm})$ increase in dive height in the optimisation to maximise height for 101B. Since elite divers should be performing with near optimum technique it is to be expected that differences between recorded performance and maximised simulation will be relatively small for the same initial conditions and the same board clearance distance. Unsurprisingly the majority of the isometric parameters came from the matching of the more demanding forward $2 \frac{1}{2}$ piked somersault dive (105B) which required more of a limiting effort at more joints. The combined set of isometric parameters obtained are appropriate for use in the torque driven model for the analysis of forward dives including maximal performances, although may not be appropriate for other types of dives (e.g. reverse dives) or jumps where quite different joint torque profiles may be used. Previous research with torque-driven simulation models with maximal voluntary torque functions based on dynamometer measurements indicates that for limiting movements full activation is achieved at a number of joints (King and Yeadon, 2004; Wilson et al., 2007). It is expected therefore that the proposed method will identify effective maximal joint torque levels for the types of skills used in their determination.

The method for determining strength parameters has the advantage that it does not require dynamometer measurements to be taken on the subject. When full effort trials can be obtained it may be preferable to take isometric and isovelocity torque measurements using a 
dynamometer as this gives a full set of subject-specific parameters which can be used in different situations. In other cases using torque / angle / angular velocity relationships from a similar sports person and scaling the isometric torque strength to the specific subject through matching performances gives a set of parameters which limit the model joint strength to realistic limits for such performances. Basing the torque parameters on isovelocity measurements taken on another individual (torque - angular velocity and torque - angle relationships) could potentially lead to poor matches between simulation and performance because the variation of angle and angular velocity dependencies of torque with different subjects is unknown. This study investigates whether this procedure could produce a suitable set of parameters. Since the percentage differences in this case $(2.6 \%$ and $3.7 \%)$ compare favourably with that obtained for the matching of running jumps using subject-specific torque data determined from dynamometer measurements (6.6\%, King et al., 2006), basing the torque parameters on those of another individual seems to be justified.

Other models of takeoffs in springboard diving (Cheng and Hubbard, 2004; Sprigings and Miller, 2004) have not attempted to carry out quantitative evaluations as conducted in this study. One reason for this may have been that these models were insufficiently complex to reproduce all of the elements that have an appreciable effect on an actual performance. It may be questioned whether the model used in this study was sufficiently complex or overly complex. In jumping activities such as diving, power is developed at the hip, knee and ankle joints (van Soest et al., 1994; Sprigings and Miller, 2004), indicating that a model of springboard diving should include segments representing the trunk, thighs, shanks, and feet. In order that the centre of pressure at the foot-board interface is not constrained to be at the toes once the heels lose contact, it is also necessary to have a two-part foot segment. Since the arms can make a substantial contribution in jumps (Dapena, 1999) the model should include a two-part arm segment representing both arms. Thus the need to include the seven segments: trunk, thigh, shank, two-part foot, upper arm and lower arm is apparent. The inherent assumption of arm and leg symmetry in this model is reasonable since the landing from the hurdle step and subsequent contact phase of takeoffs for non-twisting dives typically maintain left-right symmetry. The necessity for the inclusion of the head as an eighth segment separate from the trunk is debatable since head movement relative to the trunk is small. The use of an angle-driven elbow rather than a torque-driven elbow is debatable but it is not thought that this would influence optimum solutions greatly. The inclusion of wobbling masses within the trunk, thigh and shank segments was somewhat speculative due to the compliant nature of the springboard. The torque-driven model was found to be insensitive to the wobbling mass stiffness parameter values in the sense that when the activation time histories were re-optimised for increased stiffness, a very similar matching simulation was found with very similar activation time histories. It may be concluded that it is not necessary to include wobbling masses in models of jumping from compliant media such as diving springboards. The relatively simple extensor and flexor profiles may also be questioned. The profiles have the advantage that only a relatively small number of parameters is required and they allow for different activations between the joints as shown in Figure 7 where the ankle profile is quite different to those of the hip and knee. A more complex activation profile requiring more parameters may have improved the agreement between simulation and performance although since the agreement is already good, the benefit of any increases in complexity is questionable. There is some evidence (Figure 6) that the matching ankle torque time history rises earlier than the actual and takes longer to reach maximum activation. This is not due to a limitation in the complexity of the activation profile, however, as the matching optimisation chooses a relatively slow ramp time of $291 \mathrm{~ms}$ when it could have selected a much faster ramp time (as low as $100 \mathrm{~ms}$ ). 
The combination of the close matching of the two forward rotating dives and the small increase in height when optimised indicates that the model complexity was sufficient to produce accurate simulations and that the torque parameters used were appropriate. The model is therefore suitable for simulating springboard dives from the forward group (e.g. maximising somersault rotation). Since the model was evaluated using a dive with little rotation and a dive with maximal rotation, it may be expected that the model can be applied to any forward dive. It may be concluded that the procedure described is able to determine appropriate subject-specific strength parameters suitable for use in the simulation of maximal performances. In the future the model will be used to investigate the effect of technique and strength on optimal performance in forward dives along with investigations into the contributions to performance in forward dives.

\section{Acknowledgment}

This study was supported by the International Society of Biomechanics under the Matching Dissertation Grant Program.

\section{References}

Alexander, R.M., 1990. Optimum take-off techniques for high and long jumps. Philosophical Transactions of the Royal Society of London - Series B: 329, 3-10.

Cheng, K.B., Hubbard, M., 2004. Optimal jumping strategies from compliant surfaces: a simple model of springboard standing jumps. Human Movement Science 23, 35-48.

Corana, A., Marchesi, M., Martini, C., Ridella, S., 1987. Minimising multimodal functions of continuous variables with the "simulated annealing" algorithm. ACM Transactions on Mathematical Software 13, 262-280.

Dapena, J., 1999. A biomechanical explanation of the effect of arm actions on the vertical velocity of a standing jump. In International Society of Biomechanics XVIIth Congress Book of Abstracts, p. 100. University of Calgary.

Duncan, A., McDonagh, M.J., 2000. Stretch reflex distinguished from pre-programmed muscle activations following landing impacts in man. Journal of Physiology $526 \mathrm{Pt} 2$, 457-468.

FINA. 2009. http://www.fina.org.

Horita, T., Komi, P.V., Nicol, C., Kyrolainen, H., 2002. Interaction between pre-landing activities and stiffness regulation of the knee joint musculoskeletal system in the drop jump: implications to performance. European Journal of Applied Physiology 88, 7684.

Kane, T.R., Levinson, D.A., 1985. Dynamics: Theory and implementations. McGraw-Hill, New York.

King, M.A., Yeadon, M.R., 2002. Determining subject-specific torque parameters for use in a torque-driven simulation model of dynamic jumping. Journal of Applied Biomechanics 18, 207-217.

King, M.A., Yeadon, M.R., 2004. Maximising somersault rotation in tumbling. Journal of Biomechanics 37, 471-477.

King, M.A., Wilson, C. and Yeadon, M.R. 2006. Evaluation of a torque-driven model of jumping for height. Journal of Applied Biomechanics, 22, 264-274.

Koh, M., Jennings, L., Elliott, B., Lloyd, D., 2003. A predicted optimal performance of the Yurchenko layout vault in women's artistic gymnastics. Journal of Applied Biomechanics 19, 187-204.

Mills, C., Pain, M.T.G. and Yeadon, M.R., 2008. The influence of simulation model complexity on the estimation of internal loading in gymnastics landings. Journal of Biomechanics 41, 620-628. 
Soest, A.J. van, Bobbert, M.F., Ingen Schenau, G.J. van., 1994. A control strategy for the execution of explosive movements from varying starting positions. Journal of Neurophysiology 71, 1390-1402.

Sprigings, E., Miller, D.I., 2004. Optimal knee extension timing in springboard and platform dives from the reverse group. Journal of Applied Biomechanics 20, 275-290.

Yeadon, M.R., 1990. The simulation of aerial movement - II. A mathematical inertia model of the human body. Journal of Biomechanics 23, 67-74.

Yeadon, M.R., Atha, J., Hales, F.D., 1990. The simulation of aerial movement - IV. A computer simulation model. Journal of Biomechanics 23, 85-89.

Yeadon, M.R., Hiley, M.J., 2000. The mechanics of the backward giant circle on the high bar. Human Movement Science 19, 153-173.

Yeadon, M.R., King, M.A., 2002. Evaluation of a torque driven simulation model of tumbling. Journal of Applied Biomechanics 18, 195-206.

Yeadon, M.R., King, M.A. 2008. Computer simulation modelling in sport. In Biomechanical Evaluation of Movement in Sport and Exercise: BASES Guidelines (Eds C.J. Payton and R.M. Bartlett). Routledge. Chapter 9, 176-205.

Yeadon, M.R, King, M.A., Wilson, C., 2006a. Modelling the maximum voluntary joint torque / angular velocity relationship in human movement. Journal of Biomechanics 39, 476482.

Yeadon, M.R., Kong, P.W., King, M.A., 2006b. Parameter determination for a computer simulation model of a diver and a springboard. Journal of Applied Biomechanics 22, 167-176.

Wilson, C., Yeadon. M.R., King, M.A. 2007. Considerations that affect optimised simulation in a running jump for height. Journal of Biomechanics 40, 3155-3161. 